\title{
Sharp CFL, Discrete Kinetic Formulation and Entropic Schemes for Scalar Conservation Laws
}

\author{
Charalambos Makridakis ${ }^{1}$ and Benoît Perthame ${ }^{2}$
}

\begin{abstract}
We consider semidiscrete and fully discrete conservative finite volume schemes approximating the solution to one dimensional scalar conservation law. We show that all E-schemes are associated with a discrete kinetic formulation with a nonnegative kinetic defect measure. This construction provides an alternative proof of the discrete local entropy inequalities with simple expressions of the discrete entropy fluxes. In contrast to the known results which are restricted to CFL of the form $\lambda Q \leq 1 / 2$, our proof holds under "sharp" CFL conditions.
\end{abstract}

\section{Introduction}

We consider conservative schemes approximating the scalar conservation law

$$
\begin{gathered}
\frac{\partial}{\partial t} u+\frac{\partial}{\partial x} A(u)=0, \\
u(t=0, x)=u^{0}(x) \in L^{1} \cap L^{\infty}(\mathbb{R}) .
\end{gathered}
$$

We assume and denote

$$
A(0)=0, \quad a(\cdot)=A^{\prime}(\cdot) .
$$

As usual [6], [17], (1.1) is completed by the family of entropy inequalities; for any convex function $S$, there holds

$$
\frac{\partial}{\partial t} S(u)+\frac{\partial}{\partial x} \eta^{S}(u) \leq 0
$$

with $\eta^{S}(u)=\int_{0}^{u} S^{\prime}(\xi) a(\xi) d \xi$.

The purpose of this paper is to give new proofs under improved CFL conditions for discrete local entropy inequalities for a wide class of conservative entropic schemes for (1.1): the

\footnotetext{
${ }^{1}$ Department of Applied Mathematics, University of Crete, 71409 Heraklion-Crete, Greece and Institute of Applied and Computational Mathematics, FORTH, 71110 Heraklion - Crete, Greece, makr@math.uoc.gr

${ }^{2}$ Département de Mathématiques et Applications, UMR 855, Ecole Normale Supérieure, 45, rue d'Ulm, 75230 Paris Cedex 05, France, Benoit.Perthame@ens.fr
} 
E-Schemes (see [14]), and to investigate the connection between these schemes and the discretization of the kinetic formulation of the conservation law as introduced in [13], [12]. In fact the proofs of the local entropy inequalities follow (as in the continuous case) from the positiveness of the defect measure appearing in the discrete kinetic formulation of the schemes.

In order to describe our results we introduce the following notation for the discretization. For simplicity we take a uniform mesh, but the analysis covers the nonuniform case with appropiate modifications, cf Remark 3.5.

- $h>0$ is the uniform mesh size,

- $x_{i+1 / 2}=(i+1 / 2) h, i \in \mathbb{Z}$, are the cell interfaces,

- $C_{i}$ denotes the cell $\left(x_{i-1 / 2}, x_{i+1 / 2}\right)$,

- $\Delta t$ is the time step, $t^{n}=n \Delta t$,

- $v_{i}(t)\left(\operatorname{resp} . v_{i}^{n}\right)$ denotes the solution to the numerical scheme,

- $\lambda=\frac{\Delta t}{h}$.

The principle of the finite volume method consists in conservation approximations of the solution cell-averages

$$
\begin{array}{ll}
u_{i}(t)=\frac{1}{h_{i}} \int_{C_{i}} u(t, x) d x, & \text { (semidiscrete case) }, \\
u_{i}^{n}=\frac{1}{h_{i}} \int_{C_{i}} u\left(t^{n}, x\right) d x, & \text { (fully discrete case). }
\end{array}
$$

We study both semidiscrete and fully discrete conservative schemes based on a two point numerical flux $\mathcal{A}=\mathcal{A}(u, v)$. It is chosen so that the numerical fluxes, called below $A_{i+1 / 2}(t)$, approximate the exact fluxes $A\left(u\left(t, x_{i+1 / 2}\right)\right)$. We thus require the numerical fluxes to be consistent, i.e., $\mathcal{A}(u, u)=A(u)([8],[9],[10],[11])$. The semidiscrete scheme is defined by

$$
\begin{aligned}
h \frac{d}{d t} v_{i}(t)+A_{i+1 / 2}(t) & -A_{i-1 / 2}(t)=0, \quad i \in \mathbb{Z}, \\
v_{i}(t=0) & =v_{i}^{0} \in l^{1}(\mathbb{Z}) \quad \text { given, } \\
A_{i+1 / 2}(t) & =\mathcal{A}\left(v_{i}(t), v_{i+1}(t)\right) .
\end{aligned}
$$

The corresponding fully discrete scheme that we consider is

$$
\begin{gathered}
v_{i}^{n+1}-v_{i}^{n}+\lambda\left(A_{i+1 / 2}^{n}-A_{i-1 / 2}^{n}\right)=0, \quad i \in \mathbb{Z}, \\
v_{i}(t=0)=v_{i}^{0} \in l^{1}(\mathbb{Z}) \quad \text { given, } \\
A_{i+1 / 2}^{n}=\mathcal{A}\left(v_{i}^{n}, v_{i+1}^{n}\right),
\end{gathered}
$$

where $\mathcal{A}$ is the same numerical flux.

Local entropy inequalities. It is well known that a key property that guarantees the convergence of the schemes to the unique entropy solution of the conservation law is to satisfy a discrete 
version of the entropy inequalities associated with (1.1). Namely, we are interested in schemes (1.4) for which in-cell entropy inequalities hold, i.e. for any convex function $S$, there holds

$$
\begin{aligned}
& h \frac{d}{d t} S\left(v_{i}(t)\right)+\eta_{i+1 / 2}-\eta_{i-1 / 2} \leq 0, \\
& \eta_{i+1 / 2}=\eta\left(S ; v_{i}(t), v_{i+1}(t)\right)
\end{aligned}
$$

for some appropriate entropy discrete flux function $\eta(S ; u, v)$. For fully discrete schemes we require

$$
S\left(v_{i}^{n+1}\right)-S\left(v_{i}^{n}\right)+\lambda\left(\eta_{i+1 / 2}^{n}-\eta_{i-1 / 2}^{n}\right) \leq 0, \quad i \in \mathbb{Z}
$$

A related class of schemes are the E-schemes introduced by Osher [14] in the semidiscrete case. These are the schemes for which the Lipschitz continuous function $\mathcal{A}(u, v)$ satisfies

$$
\begin{array}{ll}
\mathcal{A}(u, v) \leq A(\xi), & \text { for } u \leq \xi \leq v, \\
\mathcal{A}(u, v) \geq A(\xi), & \text { for } v \leq \xi \leq u .
\end{array}
$$

In Osher [14] was shown that (1.6) follows from the the E-property for the flux. In the fully discrete case Tadmor [18] showed that, under certain CFL limitations, E-schemes satisfy (1.7). Tadmor's seminal approach requires to write (1.5) in its viscosity form

$$
\begin{aligned}
v_{i}^{n+1}= & v_{i}^{n}-\frac{\lambda}{2}\left(A\left(v_{i+1}^{n}\right)-A\left(v_{i-1}^{n}\right)\right) \\
& +Q_{i+1 / 2}\left(v_{i+1}^{n}-v_{i}^{n}\right)-Q_{i-1 / 2}\left(v_{i}^{n}-v_{i-1}^{n}\right)
\end{aligned}
$$

where the viscosity coefficient $Q_{i+1 / 2}$ is

$$
\begin{aligned}
& Q_{i+1 / 2}=Q\left(v_{i}^{n}, v_{i+1}^{n}\right) \\
& Q(u, v)=\lambda \frac{A(u)+A(v)-2 \mathcal{A}(u, v)}{v-u} .
\end{aligned}
$$

In [18] was shown that if $\mathcal{A}(u, v)$ satisfies the E-property and the CFL conditions

$$
\begin{aligned}
& Q_{i+1 / 2} \leq \frac{1}{2}, \\
& \lambda \max _{\xi}|a(\xi)| \leq \frac{1}{2}, .
\end{aligned}
$$

are met, then the fully discrete scheme (1.5) satisfies the in-cell entropy inequalities (1.7). Later this proof was extended to the multi-dimensional finite volume setting in [5], [1], see also [15] for an improved version. As it was already noticed in [18], (1.11) are stronger than one would like to have. Indeed, consider the two limiting cases for $Q$ that correspond to Godunov and Lax-Friedrichs schemes (denote by $Q^{G}$ and $Q^{L F}$ their numerical viscosity coefficient). Then one can check that for all E-schemes, [14], [18], $Q^{G} \leq Q$, but Godunov's scheme is known to satisfy (1.7) under the sharp CFL: $\lambda \max _{\xi}|a(\xi)| \leq 1$. In addition $Q^{L F} \equiv 1$, i.e. (1.11), is also restrictive. The reason behind this restricted CFL is the method of proof in [18] which splits the numerical cell in two half subcells and reduces the numerical viscosity of any E-scheme in a convex combination of Godunov and modified Lax-Friedrichs schemes. This splitting in two subcells avoids to analyse the interaction of waves but leads to the restricted CFL. 
In the sequel we show that indeed (1.11) can be relaxed to the "sharp" conditions

$$
\begin{aligned}
& Q_{i+1 / 2} \leq 1 \\
& \lambda \max _{\xi}|a(\xi)| \leq 1 .
\end{aligned}
$$

Our proofs do not rely on the above comparison with Godunov and modified Lax-Friedrichs, rather it is based on the kinetic formulation of E-schemes that we present in the sequel.

Kinetic formulation. To each one of the schemes considered we will associate a discrete kinetic scheme. To do that we first consider the kinetic formulation of the conservation law (1.1) introduced in [13] (see also [16])

$$
\frac{\partial}{\partial t} f(x, t, \xi)+a(\xi) \frac{\partial}{\partial x} f(x, t, \xi)=\frac{\partial}{\partial \xi} m(x, t, \xi) .
$$

Then, $f(x, t, \xi)=\chi(\xi, u(x, t))$ and $m$ is a nonnegative bounded measure with compact support with respect to $\xi$, if and only if

$$
u(x, t)=\int_{\mathbb{R}} f(x, t, \xi) d \xi
$$

is the unique entropy solution of the conservation law (1.1). The kinetic equation has incorporated all the entropy inequalities (1.3). We use the standard notation for the signed characteristic function, $a \in \mathbb{R}$,

$$
\chi(\xi, a)= \begin{cases}1 & 0<\xi \leq a, \\ -1 & a \leq \xi<0, \\ 0 & \text { otherwise } .\end{cases}
$$

For later reference note the following key property of $\chi$ that allows to derive (1.3) integrating (1.14) against $S^{\prime}(\xi) d \xi$; for all the continuous functions $S$

$$
\int \chi(\xi, a) S^{\prime}(\xi) d \xi=S(a)-S(0), \quad \forall a \in \mathbb{R} .
$$

One of the results of this paper is that, for any E-flux, an appropriate upwind discretization of the linear transport part of (1.14), provides a discrete kinetic formulation. In other words when $v_{i}(t), i \in \mathbb{Z}$ is given through (1.4), then there also holds

$$
\begin{aligned}
h \frac{\partial}{\partial t} \chi\left(\xi, v_{i}(t)\right) & +\left[a_{+}\left(\xi, v_{i}, v_{i+1}\right) \chi\left(\xi, v_{i}(t)\right)-a_{-}\left(\xi, v_{i}, v_{i+1}\right) \chi\left(\xi, v_{i+1}(t)\right)\right] \\
& -\left[a_{+}\left(\xi, v_{i-1}, v_{i}\right) \chi\left(\xi, v_{i-1}(t)\right)-a_{-}\left(\xi, v_{i-1}, v_{i}\right) \chi\left(\xi, v_{i}(t)\right)\right]=\frac{\partial}{\partial \xi} m_{i}(t, \xi),
\end{aligned}
$$

where the functions in the right hand side — called the kinetic defect measures - satisfy

$$
\begin{aligned}
& m_{i}(t, \xi)=m_{-}\left(\xi, v_{i-1}, v_{i}\right)+m_{+}\left(\xi, v_{i}, v_{i+1}\right), \quad m_{ \pm}(\cdot, u, v) \geq 0 \\
& m_{ \pm}(\cdot, u, v) \text { vanish outside of the non ordered interval }(u, v),
\end{aligned}
$$

and the numerical speeds $a_{ \pm}$, bounded by quantities of order $\frac{\partial}{\partial u} \mathcal{A}, \frac{\partial}{\partial v} \mathcal{A}$ or $a(\xi)$, satisfy

$$
\begin{aligned}
& a_{ \pm}(\xi, u, v) \geq 0, \\
& a_{+}(\xi, u, v)=\max (0, a(\xi)), \quad a_{-}(\xi, u, v)=\max (0,-a(\xi)) \quad \text { for } \xi \notin(u, v), \\
& \mathcal{A}(u, v)=\int_{\mathbb{R}} a_{+}(\xi, u, v) \chi(\xi, u) d \xi-\int_{\mathbb{R}} a_{-}(\xi, u, v) \chi(\xi, v) d \xi .
\end{aligned}
$$


Therefore a simple $\xi$ integration shows that from a formula (1.17) one derives a semidiscrete scheme (1.4), and the entropy flux in (1.6) follows by integrating (1.17) against $S^{\prime}(\xi)$

$$
\eta(S ; u, v)=\int_{\mathbb{R}} a_{+}(\xi, u, v) S^{\prime}(\xi) \chi(\xi, u) d \xi-\int_{\mathbb{R}} a_{-}(\xi, u, v) S^{\prime}(\xi) \chi(\xi, v) d \xi .
$$

At this level one can observe that, for an E-scheme, the $S$-linear entropy flux is not unique and several choices of $a_{+}, a_{-}$are possible that lead to different discrete entropy fluxes.

Notice that the most natural and simple example from this point of view is Engquist-Osher scheme [7] where (1.17) holds with

$$
\begin{aligned}
& a_{+}(\xi)=\max (0, a(\xi)), \quad a_{-}(\xi)=\max (0,-a(\xi)), \\
& \mathcal{A}_{E O}(u, v)=A_{+}(u)+A_{-}(v), \\
& A_{+}(u)=\int_{0}^{u} a_{+}(\xi) d \xi, \quad A_{-}(u)=\int_{0}^{u} a_{-}(\xi) d \xi .
\end{aligned}
$$

This case has the remarkable property that the discrete kinetic formulation (1.17) is a linear equation on $\chi$, a fundamental property in the continuous formulation (1.14) which allows for instance a convergence proof of Engquist-Osher scheme based on merely $L^{\infty}$ bounds (see [2]). This simple case is also a model for kinetic schemes for systems of conservation laws ([3], [19], [16]) and allows to give another convergence proof [20]. An alternative proof based on the framework of [13] and a kinetic formulation of Godunov's finite volume scheme was given in $[21]$.

To recover fully discrete schemes (1.5) by a kinetic formulation is more intricate and therefore we may have to introduce more general discetizations of the linear transport part of (1.14). We thus define,

Definition 1.1. The function $a(\xi, u, v)$ which is integrable and has compact support with respect to $\xi$ is called a Discrete Kinetic Flux corresponding to $\mathcal{A}(u, v)$ if

$$
\begin{aligned}
& \int_{\mathbb{R}} a(\xi, u, v) d \xi=\mathcal{A}(u, v), \\
& a(\xi, u, u)=a(\xi) \chi(\xi, u)=A^{\prime}(\xi) \chi(\xi, u) .
\end{aligned}
$$

In the semi-discrete case our choice can be e.g., $a(\xi, u, v)=a_{+}(\xi, u, v) \chi(\xi, u)+a_{-}(\xi, u, v) \chi(\xi, v)$ but in the fully dicrete case we have to consider more general representation formulas.

In Section 2 we investigate the semidiscrete scheme (1.4) and we prove in Theorem 2.1, that E-schemes are characterized by the existence of a semidiscrete kinetic formulations (1.17). In fact we show first that the existence of a more general discrete kinetic formulation, cf. (2.1), is equivalent to the fact that $\mathcal{A}(u, v)$ is an E-flux. Towards this goal a crucial step is that the integrant of the Discrete Kinetic Flux, defined in (2.8), should satisfy the requirements provided by Lemma 2.4 and further by Proposition 2.6.

In Section 3 we investigate the fully discrete scheme (1.5) and the existence of a Discrete Kinetic Flux corresponding to $\mathcal{A}(u, v), a(\xi, u, v)$, such that

$$
\begin{aligned}
\chi\left(\xi, v_{i}^{n+1}\right)-\chi\left(\xi, v_{i}^{n}\right) & +\lambda\left[a\left(\xi, v_{i}^{n}, v_{i+1}^{n}\right)-a\left(\xi, v_{i-1}^{n}, v_{i}^{n}\right)\right] \\
& =\frac{\partial}{\partial \xi} m_{i}^{n}(\xi),
\end{aligned}
$$


with $m_{i}^{n}$ a nonnegative function as in (1.18). Our main result is that if $\mathcal{A}(u, v)$ is an E-flux and the CFL conditions (1.13) are met then we can construct $a(\xi, u, v)=a_{\lambda}(\xi, u, v)$ such that (1.23) is a kinetic formulation of (1.5) with $m_{i}^{n}$ nonnegative, Theorem 3.1. Then the in-cell entropy inequalities (1.7) follow with entropy flux $\eta_{i+1 / 2}=\eta(\lambda, S ; u, v)=\int_{\mathbb{R}} S^{\prime}(\xi) a_{\lambda}(\xi, u, v) d \xi$. The proof is constructive and the conditions on $a(\xi, u, v)$ derived in Section 2 for the semidiscrete problem are particularly useful in the analysis.

In Section 4 we give the construction, and additional explicit formulas, for Engquist-Osher scheme. This section can be viewed as a model for the generic construction in Section 2 .

\section{Semidiscrete schemes}

In this section we investigate general three point semidiscrete scheme (1.4) with consistent flux $\mathcal{A}(u, v)$. We prove the equivalence between three properties; the E-property, the possibility to write a kinetic discretization as (1.17), and the existence of discrete entropy fluxes in (1.6).

Namely, the main result of this section is the

Theorem 2.1. Consider the semidiscrete scheme (1.4) with a consistent discrete flux $\mathcal{A}(u, v)$. The following three properties are equivalent

(i) $\mathcal{A}(u, v)$ is an E-flux as defined in (1.8);

(ii) all the in-cell entropy inequalities (1.6), i.e. for any convex function $S$, are satisfied

(iii) there exists a Discrete Kinetic Flux $a(\xi, u, v)$ corresponding to $\mathcal{A}(u, v)$, and nonnegative functions $m_{i}$ satisfying (1.18), such that the kinetic formulation of (1.4) holds

$$
h \frac{\partial}{\partial t} \chi\left(\xi, v_{i}(t)\right)+\left[a\left(\xi, v_{i}, v_{i+1}\right)-a\left(\xi, v_{i-1}, v_{i}\right)\right]=\frac{\partial}{\partial \xi} m_{i}(t, \xi) .
$$

The entropy fluxes in (1.6), as well as $a(\xi, u, v)$, is not unique and a possible relation is

$$
\eta(S ; u, v)=\int_{\mathbb{R}} S^{\prime}(\xi) a(\xi, u, v) d \xi .
$$

In addition $a(\xi, u, v)$ admits an "upwind" splitting of the form (1.19).

We first recall the equivalence between properties (i) and (ii) for the sake of completeness. The property (iii) is then derived in several steps. We conclude this section with an explicit construction of Discrete Kinetic Fluxes like (1.19).

Proof of Theorem 2.1, (i) $\Leftrightarrow$ (ii). We depart from (ii). Multiplying (1.4) by $S^{\prime}\left(v_{i}(t)\right.$ ), we obtain that the in-cell entropy inequality is equivalent to the existence of $\eta(S, \cdot, \cdot)$ such that, for all values $v_{i}, v_{i \pm 1}$ and all convex $S$, we have

$$
\eta\left(S ; v_{i}, v_{i+1}\right)-\eta\left(S ; v_{i-1}, v_{i}\right) \leq S^{\prime}\left(v_{i}\right)\left[\mathcal{A}\left(v_{i}, v_{i+1}\right)-\mathcal{A}\left(v_{i-1}, v_{i}\right)\right],
$$

which is equivalent to

$$
\left\{\begin{array}{l}
\eta\left(S ; v_{i}, v_{i+1}\right)-\eta\left(S ; v_{i}, v_{i}\right) \leq S^{\prime}\left(v_{i}\right)\left[\mathcal{A}\left(v_{i}, v_{i+1}\right)-A\left(v_{i}\right)\right] \\
\eta\left(S ; v_{i}, v_{i}\right)-\eta\left(S ; v_{i-1}, v_{i}\right) \leq S^{\prime}\left(v_{i}\right)\left[A\left(v_{i}\right)-\mathcal{A}\left(v_{i-1}, v_{i}\right)\right]
\end{array}\right.
$$


which is again equivalent, for all $u, v$, and $S$ convex, to the existence of a function $\eta(S, \cdot, \cdot)$ such that

$$
\eta(S ; v, v)-S^{\prime}(v)[A(v)-\mathcal{A}(u, v)] \leq \eta(S ; u, v) \leq S^{\prime}(u)[\mathcal{A}(u, v)-A(u)]+\eta(S ; u, u) .
$$

Denoting $\eta(S ; v)=\eta(S ; v, v)$, the above inequality is obviously equivalent to (and then the choice of $\eta(S ; u, v)$ is anything in-between)

$$
\eta(S ; v)-S^{\prime}(v)[A(v)-\mathcal{A}(u, v)] \leq S^{\prime}(u)[\mathcal{A}(u, v)-A(u)]+\eta(S ; u),
$$

or, in other words,

$$
\eta(S ; v)-\eta(S ; u) \leq S^{\prime}(v) A(v)-S^{\prime}(u) A(u)-\mathcal{A}(u, v)\left[S^{\prime}(v)-S^{\prime}(u)\right],
$$

which is equivalent to

$$
\left\{\begin{array}{l}
\frac{\partial}{\partial u} \eta(S ; u)=S^{\prime}(u) a(u) \\
\int_{v}^{u} S^{\prime \prime}(\zeta) A(\zeta) d \zeta \leq \mathcal{A}(u, v)\left[S^{\prime}(u)-S^{\prime}(v)\right]
\end{array}\right.
$$

and it remains to choose, as a generating family for $S$ convex, the family $S^{\prime \prime}(\zeta)=\delta(\zeta-\xi)$ to recover the equivalence with the E-property (1.8).

We would like to conclude with noticing that the entropy fluxes are automatically consistent, i.e. the relation $\frac{\partial}{\partial u} \eta(S ; u, u)=S^{\prime}(u) a(u)$ is derived from (ii).

We now introduce some steps towards the semidiscrete kinetic formulation (iii). We start with

Lemma 2.1. Let $a(\xi, u, v)$ be a Discrete Kinetic Flux corresponding to $\mathcal{A}(u, v)$, we have

$$
m_{i}(t, \xi)=m_{+}\left(\xi ; v_{i}, v_{i+1}\right)+m_{-}\left(\xi ; v_{i-1}, v_{i}\right),
$$

with

$$
\begin{aligned}
& m_{+}(\xi ; u, v)=\int_{-\infty}^{\xi} \delta(\zeta-u)[A(u)-\mathcal{A}(u, v)] d \zeta+\int_{-\infty}^{\xi}[a(\zeta, u, v)-a(\zeta) \chi(\zeta, u)] d \zeta \\
& m_{-}(\xi ; u, v)=-\int_{-\infty}^{\xi} \delta(\zeta-v)[A(v)-\mathcal{A}(u, v)] d \zeta-\int_{-\infty}^{\xi}[a(\zeta, u, v)-a(\zeta) \chi(\zeta, v)] d \zeta .
\end{aligned}
$$

Moreover $m_{i}$ is nonnegative for any value of its arguments if and only if both $m_{+}$and $m_{-}$are nonnegative for any value of their arguments.

Proof. It is a simple matter to check that

$$
\frac{\partial}{\partial t} \chi\left(\xi, v_{i}(t)\right)=\delta\left(\xi-v_{i}(t)\right) \frac{d}{d t} v_{i}(t) .
$$

Then using the above formula and (1.4) in (2.1), we get

$$
\begin{aligned}
-\delta\left(\xi-v_{i}(t)\right)\left[A_{i+1 / 2}-A_{i-1 / 2}\right] & +\left[a\left(\xi, v_{i}(t), v_{i+1}(t)\right)-a\left(\xi, v_{i-1}(t), v_{i}(t)\right)\right] \\
& =\frac{\partial}{\partial \xi} m_{i}(\xi, t),
\end{aligned}
$$


or equivalently,

$$
\begin{aligned}
\frac{\partial}{\partial \xi} m_{i}(\xi, t)= & \delta\left(\xi-v_{i}(t)\right)\left[\left(A\left(v_{i}(t)\right)-A_{i+1 / 2}\right)-\left(\left(A\left(v_{i}(t)\right)-A_{i-1 / 2}\right)\right]\right. \\
& +\left[a\left(\xi, v_{i}(t), v_{i+1}(t)\right)-a(\xi) \chi\left(\xi, v_{i}(t)\right)\right] \\
& -\left[a\left(\xi, v_{i-1}(t), v_{i}(t)\right)-a(\xi) \chi\left(\xi, v_{i}(t)\right)\right]
\end{aligned}
$$

Since we want $m_{i}$ to have bounded support, we can integrate to obtain,

$$
\begin{aligned}
m_{i}(\xi, t)= & \int_{-\infty}^{\xi} \delta\left(\zeta-v_{i}(t)\right)\left[\left(A\left(v_{i}(t)\right)-A_{i+1 / 2}\right)-\left(\left(A\left(v_{i}(t)\right)-A_{i-1 / 2}\right)\right] d \zeta\right. \\
& +\int_{-\infty}^{\xi}\left[a\left(\zeta, v_{i}(t), v_{i+1}(t)\right)-a(\zeta) \chi\left(\zeta, v_{i}(t)\right)\right] d \zeta \\
& -\int_{-\infty}^{\xi}\left[a\left(\zeta, v_{i-1}(t), v_{i}(t)\right)-a(\zeta) \chi\left(\zeta, v_{i}(t)\right)\right] d \zeta \\
= & m_{+}\left(\xi, v_{i}(t), v_{i+1}(t)\right)+m_{-}\left(\xi, v_{i-1}(t), v_{i}(t)\right) .
\end{aligned}
$$

By the definition of the discrete kinetic fluxes (Definition 1.1) and the consistency of the flux $\mathcal{A}(u, v)$ we see that

$$
m_{+}(\xi, v, v)=0, \quad m_{-}(\xi, v, v)=0,
$$

thus $m$ is nonnegative if and only if both $m_{+}$and $m_{-}$are nonnegative.

Remark 2.2. Since $m_{ \pm}(+\infty ; u, v)=m_{ \pm}(-\infty ; u, v)=0$ we can see that (2.3) takes the form

$$
\begin{aligned}
& m_{+}(\xi ; u, v)=\int_{-\infty}^{\xi}[a(\zeta, u, v)-a(\zeta) \chi(\zeta, u)] d \zeta \quad \text { for } \quad \xi<u \\
& m_{+}(\xi ; u, v)=-\int_{\xi}^{+\infty}[a(\zeta, u, v)-a(\zeta) \chi(\zeta, u)] d \zeta \quad \text { for } \quad u<\xi \\
& m_{-}(\xi ; u, v)=-\int_{-\infty}^{\xi}[a(\zeta, u, v)-a(\zeta) \chi(\zeta, v)] d \zeta \quad \text { for } \quad \xi<v \\
& m_{-}(\xi ; u, v)=\int_{\xi}^{+\infty}[a(\zeta, u, v)-a(\zeta) \chi(\zeta, v)] d \zeta \quad \text { for } \quad v<\xi
\end{aligned}
$$

We proceed by further reducing the form of $m_{i}$. We need some more notation. For $u, v \in \mathbb{R}$ we denote by $I_{u, v}$ the interval that they define. Also,

$$
I_{u, v}=[m, M], \quad \text { where } m=\min \{u, v\} \text {, and } M=\max \{u, v\} .
$$

We then notice the idendity

$$
\chi(\xi, u)=\chi(\xi, v) \quad \text { for } \xi \in \mathbb{R} \backslash I_{u, v} .
$$

Then one may check, 
Lemma 2.3. Assume that $a(\xi, u, v)$ is a Discrete Kinetic Flux corresponding to $\mathcal{A}(u, v)$. If $m_{+}$and $m_{-}$are both nonnegative, then $a(\xi, u, v)$ satisfies the consistency condition outside the interval $I_{u, v}$ :

$$
a(\xi, u, v)=a(\xi) \chi(\xi, u)=a(\xi) \chi(\xi, v) \quad \xi \in \mathbb{R} \backslash I_{u, v} .
$$

Conversely, if (2.7) is satisfied then $m_{+}$and $m_{-}$vanish (and therefore are nonnegative) outside the interval $I_{u, v}$.

Proof. Assume first that $\xi<m<0$ then $\chi(\xi, u)=\chi(\xi, v)=0$. In addition both $m_{+}$and $m_{-}$ are nonnegative therefore (2.4) implies that

$$
\int_{-\infty}^{\xi} a(\zeta, u, v) d \zeta=0
$$

Since $\xi$ is arbitrary $a(\xi, u, v)=0$ for $\xi<m<0$. Similarly, if $\xi>M>0, a(\xi, u, v)=0$. In the case where $M<0$ and $M<\xi$ again by (2.4) we have

$$
\int_{\xi}^{+\infty}[a(\zeta, u, v)-a(\zeta) \chi(\zeta, u)] d \zeta=0 .
$$

Since $M<\xi$ is arbitrary we conclude that $a(\xi, u, v)=a(\xi) \chi(\xi, u)=a(\xi) \chi(\xi, v)$. The proof is similar in the case $\xi<m, m>0$.

In view of Lemma 2.3 we are able to define a function $\mathcal{A}(\xi, u, v)$ of three variables as

$$
\mathcal{A}(\xi, u, v)=\int_{-\infty}^{\xi} a(\zeta, u, v) d \zeta
$$

It is to be noted that $\mathcal{A}(\xi, u, v)$ should not be confused with the discrete flux $\mathcal{A}(u, v)$, although they are of course related depending on the values of $\xi$ since by Definition 1.1

$$
\mathcal{A}(+\infty, u, v)=\mathcal{A}(u, v) .
$$

In the next lemma we derive conditions for the Discrete Kinetic Flux in $I_{u, v}$ by using its integrant $\mathcal{A}(\xi, u, v)$.

Lemma 2.4. Assume that $a(\xi, u, v)$ is a Discrete Kinetic Flux corresponding to $\mathcal{A}(u, v)$ and $\mathcal{A}(\xi, u, v)$ is defined by (2.8). Let $m_{+}$and $m_{-}$be both nonnegative. Then the following conditions are satisfied in the interval $I_{u, v}$

$$
u \leq \xi \leq v \quad\left\{\begin{array}{l}
A(\xi) \geq \mathcal{A}(\xi, u, v) \geq \mathcal{A}(u, v), \quad \text { when } \xi \geq 0 \\
0 \geq \mathcal{A}(\xi, u, v) \geq \mathcal{A}(u, v)-A(\xi), \quad \text { when } \xi<0
\end{array}\right.
$$

and

$$
v \leq \xi \leq u \quad\left\{\begin{array}{l}
\mathcal{A}(u, v) \geq \mathcal{A}(\xi, u, v) \geq A(\xi), \quad \text { when } \xi \geq 0, \\
\mathcal{A}(u, v)-A(\xi) \geq \mathcal{A}(\xi, u, v) \geq 0, \quad \text { when } \xi<0,
\end{array}\right.
$$

Conversely, if (2.9), (2.10) are satisfied then $m_{+}$and $m_{-}$are nonnegative in the interval $I_{u, v}$. 
Proof. We only treat the case $u<\xi<v$ since the other is similar. Then, equations (2.3) imply

$$
\begin{gathered}
m_{+}(\xi, u, v)=A(u)-\mathcal{A}(u, v)+\mathcal{A}(\xi, u, v)-\int_{-\infty}^{\xi} a(\zeta) \chi(\zeta, u) d \zeta \\
m_{-}(\xi, u, v)=-\mathcal{A}(\xi, u, v)+\int_{-\infty}^{\xi} a(\zeta) \chi(\zeta, v) d \zeta
\end{gathered}
$$

But then it is easy to check that

$$
-\int_{-\infty}^{\xi} a(\zeta) \chi(\zeta, u) d \zeta=A(\xi) \mathbb{I}_{\{\xi<0\}}-A(u),
$$

and

$$
\int_{-\infty}^{\xi} a(\zeta) \chi(\zeta, v) d \zeta=A(\xi) \mathbb{I}_{\{\xi>0\}}
$$

Since both $m_{+}$and $m_{-}$should be nonnegative (2.9) follows. The converse is also immediate by using the above identities.

Remark 2.5. From Lemma 2.3 we deduce that both $m_{+}$and $m_{-}$are supported in $I_{u, v}$. Further by the proof of the Lemma 2.4 we have the following formulas

$$
\begin{aligned}
& m_{+}(\xi ; u, v)=\mathcal{A}(\xi, u, v)-\mathcal{A}(u, v)+A(\xi) \mathbb{I}_{\{\xi<0\}}, \\
& u \leq \xi \leq v: \\
& m_{-}(\xi ; u, v)=A(\xi) \mathbb{I}_{\{\xi>0\}}-\mathcal{A}(\xi, u, v),
\end{aligned}
$$

and

$$
\begin{array}{ll}
v \leq \xi \leq u: & m_{+}(\xi ; u, v)=\mathcal{A}(\xi, u, v)-A(\xi) \mathbb{I}_{\{\xi>0\}}, \\
& m_{-}(\xi ; u, v)=\mathcal{A}(u, v)-\mathcal{A}(\xi, u, v)-A(\xi) \mathbb{I}_{\{\xi<0\}} .
\end{array}
$$

We have now the following result.

Proposition 2.6. Assume that we have at our disposal a Lipschitz function $\mathcal{A}(\xi, u, v)$ which satisfies (2.9) and (2.10) and the endpoint values

$$
\text { for } u \leq v \quad\left\{\begin{array}{l}
\mathcal{A}(u, u, v)=A(u) \mathbb{I}_{\{u>0\}}, \\
\mathcal{A}(v, u, v)=\mathcal{A}(u, v)-A(v) \mathbb{I}_{\{v<0\}}
\end{array}\right.
$$

and

$$
\text { for } v \leq u \quad\left\{\begin{array}{l}
\mathcal{A}(u, u, v)=A(u, v)-A(u) \mathbb{I}_{\{u<0\}} \\
\mathcal{A}(v, u, v)=A(v) \mathbb{I}_{\{v>0\}}
\end{array}\right.
$$

Then $a(\xi, u, v)$ is well defined by

$$
\begin{aligned}
& a(\xi, u, v)=\frac{\partial}{\partial \xi} \mathcal{A}(\xi, u, v) \quad \xi \in I_{u, v} \\
& a(\xi, u, v)=a(\xi) \chi(\xi, u)=a(\xi) \chi(\xi, v) \quad \xi \in \mathbb{R} \backslash I_{u, v} .
\end{aligned}
$$

In addition $a(\xi, u, v)$ is a Discrete Kinetic Flux corresponding to $\mathcal{A}(u, v)$ and (2.1) is a kinetic formulation of (1.4) with $m_{i}$ nonnegative. 
Proof. Having (2.8) and (2.6) in mind, we first extend $\mathcal{A}(\xi, u, v)$ outside the interval $I_{u, v}$ by letting

$$
\mathcal{A}(\xi, u, v)=\int_{-\infty}^{\xi} a(\zeta) \chi(\zeta, u) d \zeta, \quad \xi \leq m
$$

and

$$
\mathcal{A}(\xi, u, v)=\int_{-\infty}^{m} a(\zeta) \chi(\zeta, u) d \zeta+A(M, u, v)-A(m, u, v)+\int_{M}^{\xi} a(\zeta) \chi(\zeta, u) d \zeta, \quad \xi \geq M .
$$

Then the function $\mathcal{A}(\cdot, u, v)$, is a well defined, continuous function and $a(\xi, u, v)$ is the derivative of $\mathcal{A}(\xi, u, v), \xi \in \mathbb{R}$. Then it is easy to see that since $\mathcal{A}(\xi, u, v)$ satisfies (2.9) and (2.10) with equalities at the endpoints of the interval $I_{u, v}$,

$$
\mathcal{A}(+\infty, u, v)=\int_{\mathbb{R}} a(\xi, u, v) d \xi=A(u, v),
$$

i.e., $a(\xi, u, v)$ is a Discrete Kinetic Flux corresponding to $\mathcal{A}(u, v)$. The proof is complete in view of Lemmas 2.3 and 2.4 .

We are now ready to complete the proof the last equivalence in Theorem 2.1.

Proof of Theorem 2.1, (i) $\Leftrightarrow$ (iii). Assume first (i), i.e. that $\mathcal{A}(u, v)$ is an E-flux. Then one can construct a discrete kinetic flux as in Lemma 2.4 and Proposition 2.6. Indeed, one choice of $\mathcal{A}(\xi, u, v)$ in $I_{u, v}$ is

$$
\text { for } u \leq \xi \leq v: \quad \mathcal{A}(\xi, u, v)=\max \{\mathcal{A}(u, \xi), \mathcal{A}(u, v)\}-A(\xi) \mathbb{I}_{\{\xi<0\}},
$$

and

$$
\text { for } v \leq \xi \leq u: \quad \mathcal{A}(\xi, u, v)=\min \{\mathcal{A}(\xi, v), \mathcal{A}(u, v)\}-A(\xi) \mathbb{I}_{\{\xi<0\}}
$$

Then since $\mathcal{A}$ is an E-flux is is straightforward to verify that $\mathcal{A}(\xi, u, v)$ satisfies $(2.9)$ and (2.10) with equalities at the endpoints of the interval $I_{u, v}$. Therefore Proposition 2.6 implies that (iii) holds.

Conversely, if (iii) holds with $m$ nonnegative then $\mathcal{A}(\xi, u, v)$ defined in (2.8) should satisfy (2.9) and (2.10). But then necessarily $\mathcal{A}(u, v)$ satisfies,

$$
\begin{array}{ll}
\text { if } u \leq \xi \leq v: & A(\xi) \geq \mathcal{A}(u, v), \\
\text { if } v \leq \xi \leq u: & \mathcal{A}(u, v) \geq A(\xi),
\end{array}
$$

i.e., $\mathcal{A}(u, v)$ is an E-flux and (i) is proved.

End of the proof of Theorem 2.1. I remains to consider another choice in Lemma 2.4 and Proposition 2.6 in order to obtain the refined kinetic formulation (1.17) with signed speeds. We built an admissible (i.e that satisfies (2.9), (2.10), (2.17), (2.18)) Lipschitz function $\mathcal{A}(\xi, u, v)$ which is nonincreasing in $\xi$ for $u<v$ and increasing in $\xi$ for $v<u$. We give the formula and skip the tedious but easy proof.

$$
u \leq \xi \leq v: \quad \mathcal{A}(\xi, u, v)=\left\{\begin{array}{l}
\max \left\{\mathcal{A}(u, v), \min _{\max (0, u) \leq \zeta \leq \xi} \mathcal{A}(u, \zeta)\right\} \quad \text { when } \xi \geq 0, \\
\max _{\xi \leq \zeta \leq \min (0, v)}\{\max (\mathcal{A}(u, v), \mathcal{A}(u, \zeta))-A(\zeta)\} \quad \text { when } \xi<0
\end{array}\right.
$$




$$
v \leq \xi \leq u: \quad \mathcal{A}(\xi, u, v)=\left\{\begin{array}{l}
\min \left\{\mathcal{A}(u, v), \max _{\max (0, v) \leq \zeta \leq \xi} \mathcal{A}(\zeta, v)\right\} \quad \text { when } \xi \geq 0, \\
\min _{\xi \leq \zeta \leq \min (0, u)}\{\min (\mathcal{A}(u, v), \mathcal{A}(\zeta, v))-A(\zeta)\} \quad \text { when } \xi<0 .
\end{array}\right.
$$

Thanks to the monotonicity of $\mathcal{A}(\xi, u, v)$, one readily checks the that indeed (1.17) holds with

$$
\begin{gathered}
a_{ \pm}(\xi, u, v)=\left|\frac{\partial}{\partial \xi} \mathcal{A}(\xi, u, v)\right| \quad \text { for } \xi \in I_{u, v}, \\
a_{ \pm}(\xi, u, v)=a_{ \pm}(\xi) \quad \text { for } \xi \in \mathbb{R} \backslash I_{u, v} .
\end{gathered}
$$

\section{$3 \quad$ Fully discrete schemes}

For a given fully discrete scheme (1.5) we will associate a discrete kinetic formulation as follows. Assume that we are given approximations at level $n: v_{i}^{n}, i \in \mathbb{Z}$. Define then the approximations at the next level as

$$
\begin{aligned}
f_{i}^{n+1} & =\chi\left(\xi, v_{i}^{n}\right)-\lambda\left[a\left(\xi, v_{i}^{n}, v_{i+1}^{n}\right)-a\left(\xi, v_{i-1}^{n}, v_{i}^{n}\right)\right] \quad \text { and } \\
v_{i}^{n+1} & =\int f_{i}^{n+1}(\xi) d \xi .
\end{aligned}
$$

We call (3.1) a kinetic formulation of the difference scheme $(1.5)$ if $a(\xi, u, v)$ is a Discrete Kinetic Flux corresponding to $\mathcal{A}(u, v)$ and there exist measures with compact support with respect to $\xi, m_{i}^{n}$, such that

$$
\chi\left(\xi, v_{i}^{n+1}\right)-f_{i}^{n+1}=\frac{\partial}{\partial \xi} m_{i}^{n}(\xi) .
$$

Then integrating (3.2) with respect to $\xi$ we recover the scheme (1.5). In such a case the discrete kinetic scheme can be written in a compact form as

$$
\begin{aligned}
\chi\left(\xi, v_{i}^{n+1}\right)-\chi\left(\xi, v_{i}^{n}\right) & +\lambda\left[a\left(\xi, v_{i}^{n}, v_{i+1}^{n}\right)-a\left(\xi, v_{i-1}^{n}, v_{i}^{n}\right)\right] \\
& =\frac{\partial}{\partial \xi} m_{i}^{n}(\xi) .
\end{aligned}
$$

In this section we will investigate under what conditions on $\mathcal{A}(u, v)$ and $a(\xi, u, v)$ the scheme (3.1) is a kinetic formulation of (1.5) with nonnegative $m_{i}^{n}(\xi)$, i.e., under what conditions (3.3) holds with $m_{i}^{n}(\xi)$ nonnegative. This will imply that the scheme satisfies all local discrete entropy inequalities:

Theorem 3.1. Consider a conservative scheme (1.5) with a consistent discrete flux $\mathcal{A}=\mathcal{A}(u, v)$. Assume the following

(i) $\mathcal{A}(u, v)$ is an E-flux;

(ii) the CFL condition (1.13) is satisfied. 
Then there exists a Discrete Kinetic Flux corresponding to $\mathcal{A}(u, v), a=a_{\lambda}(\xi, u, v)$, and a nonnegative measure $m$ such that (3.3) is a kinetic formulation of (1.5). Consequently, all the in-cell entropy inequalities, i.e. for any convex function $S$, hold true

$$
S\left(v_{i}^{n+1}\right)-S\left(v_{i}^{n}\right)+\lambda\left[\eta_{i+1 / 2}^{n}-\eta_{i-1 / 2}^{n}\right] \leq 0,
$$

with discrete entropy flux

$$
\eta_{i+1 / 2}^{n}=\eta\left(S ; v_{i}^{n}, v_{i+1}^{n}\right) \quad \eta(S ; u, v)=\int_{\mathbb{R}} S^{\prime}(\xi) a(\xi, u, v) d \xi .
$$

Remark 3.1. In our construction, the Discrete Kinetic Flux depends on $\lambda$. Especially we do not answer the open question to know wether, for E-schemes and under the CFL condition (1.13), there are in-cell entropy inequalities with $\eta(S)$ independent of $\lambda$. Because of this difference, it seems that a reverse theorem is wrong; the existence of a fully discrete kinetic formulation with $a_{\lambda}$, or of in-cell entropy inequalities with $\eta_{\lambda}(S)$, do not imply the E-property. Note that still in the construction of [18] the discrete entropy flux depends on $\lambda$. We recall that a weaker property, called 'ordered schemes' (restrict the E-property to $\xi=u$ or $v$ ), is enough to have a TVD scheme, see [16].

As in the previous section, for $u, v \in \mathbb{R}$ we denote by $I_{u, v}$ the interval that they define. We will need the following lemmas.

Lemma 3.2. Let $a(\xi, u, v)$ be a Discrete Kinetic Flux corresponding to $\mathcal{A}(u, v)$, and assume that (3.3) is a kinetic formulation of (1.5). Setting

$$
I_{v_{i}^{n}, v_{i}^{n+1}}=[m, M], \quad \text { where } m=\min \left\{v_{i}^{n}, v_{i}^{n+1}\right\}, \text { and } M=\max \left\{v_{i}^{n}, v_{i}^{n+1}\right\},
$$

we have for $\xi \in \mathbb{R} \backslash I_{v_{i}^{n}, v_{i}^{n+1}}$ :

$$
\begin{aligned}
& m\left(\xi ; v_{i-1}^{n}, v_{i}^{n}, v_{i+1}^{n}\right)=\lambda \int_{-\infty}^{\xi}\left[a\left(\zeta, v_{i}^{n}, v_{i+1}^{n}\right)-a\left(\zeta, v_{i-1}^{n}, v_{i}^{n}\right)\right] d \zeta \quad \text { for } \quad \xi<m \\
& m\left(\xi ; v_{i-1}^{n}, v_{i}^{n}, v_{i+1}^{n}\right)=-\lambda \int_{\xi}^{+\infty}\left[a\left(\zeta, v_{i}^{n}, v_{i+1}^{n}\right)-a\left(\zeta, v_{i-1}^{n}, v_{i}^{n}\right)\right] d \zeta \quad \text { for } \quad M<\xi
\end{aligned}
$$

Proof. Assume first that $\xi<m<0$ then $\chi\left(\xi, v_{i}^{n+1}\right)=\chi\left(\xi, v_{i}^{n}\right)=0$. Also if $\xi<m$ and $m>0$ then for $\xi<0, \chi\left(\xi, v_{i}^{n+1}\right)=\chi\left(\xi, v_{i}^{n}\right)=0$ and for $\xi>0, \chi\left(\xi, v_{i}^{n+1}\right)=\chi\left(\xi, v_{i}^{n}\right)=1$. Therefore

$$
\int_{-\infty}^{\xi} \chi\left(\zeta, v_{i}^{n+1}\right)-\chi\left(\zeta, v_{i}^{n}\right) d \zeta=0, \quad \text { for } \xi<m
$$

Similarly, we show

$$
\left.\int_{-\infty}^{\xi} \chi\left(\zeta, v_{i}^{n+1}\right)-\chi\left(\zeta, v_{i}^{n}\right) d \zeta=v_{i}^{n+1}\right)-v_{i}^{n}, \quad \text { for } \xi>M,
$$

and therefore (3.5) follows in view of (1.5) and Definition 1.1. 
Lemma 3.3. Under the assumptions of Lemma 3.2 we have for $\xi \in I_{v_{i}^{n}, v_{i}^{n+1}}$ :

$$
\begin{aligned}
& m\left(\xi ; v_{i-1}^{n}, v_{i}^{n}, v_{i+1}^{n}\right)=\xi-v_{i}^{n}+\lambda \int_{-\infty}^{\xi}\left[a\left(\zeta, v_{i}^{n}, v_{i+1}^{n}\right)-a\left(\zeta, v_{i-1}^{n}, v_{i}^{n}\right)\right] d \zeta \quad \text { for } \quad v_{i}^{n}<\xi \\
& m\left(\xi ; v_{i-1}^{n}, v_{i}^{n}, v_{i+1}^{n}\right)=v_{i}^{n}-\xi-\lambda \int_{\xi}^{+\infty}\left[a\left(\zeta, v_{i}^{n}, v_{i+1}^{n}\right)-a\left(\zeta, v_{i-1}^{n}, v_{i}^{n}\right)\right] d \zeta \quad \text { for } \quad \xi<v_{i}^{n}
\end{aligned}
$$

Proof. Assume first that $v_{i}^{n}<\xi<v_{i}^{n+1}$. Then one can verify that,

$$
\int_{-\infty}^{\xi} \chi\left(\zeta, v_{i}^{n+1}\right)-\chi\left(\zeta, v_{i}^{n}\right) d \zeta=\xi-v_{i}^{n}
$$

which implies the first equality of (3.6). Similarly, there holds,

$$
\int_{-\infty}^{\xi} \chi\left(\zeta, v_{i}^{n+1}\right)-\chi\left(\zeta, v_{i}^{n}\right) d \zeta=v_{i}^{n+1}-\xi, \quad \text { for } v_{i}^{n+1}<\xi<v_{i}^{n}
$$

and in this case (3.6) follows again in view of (1.5) and Definition 1.1.

We are ready now to prove the main result in this section.

Proof of Theorem 3.1. For the given discrete flux $\mathcal{A}(u, v)$ we first observe that if $a(\xi, u, v)$ is a function that is constructed according to Proposition 2.6, i.e. if $a(\xi, u, v)$ is a kinetic flux for the semidiscrete scheme, then $m\left(\xi ; v_{i-1}^{n}, v_{i}^{n}, v_{i+1}^{n}\right)$ in Lemma 3.2 are nonnegative. Indeed if $\xi<m$ then $\xi<v_{i}^{n}$ and

$$
m\left(\xi ; v_{i-1}^{n}, v_{i}^{n}, v_{i+1}^{n}\right)=m_{+}\left(\xi ; v_{i}^{n}, v_{i+1}^{n}\right)+m_{-}\left(\xi ; v_{i-1}^{n}, v_{i}^{n}\right)
$$

where $m_{+}$and $m_{-}$are defined in (2.4) for $\xi<v_{i}^{n}$. A similar relation holds for $\xi>M$. The same reasoning implies that

$$
\int_{-\infty}^{z}\left[a\left(\zeta, v_{i}^{n}, v_{i+1}^{n}\right)-a\left(\zeta, v_{i-1}^{n}, v_{i}^{n}\right)\right] d \zeta \geq 0 \quad \text { for all } \quad z<v_{i}^{n}
$$

and

$$
-\int_{z}^{+\infty}\left[a\left(\zeta, v_{i}^{n}, v_{i+1}^{n}\right)-a\left(\zeta, v_{i-1}^{n}, v_{i}^{n}\right)\right] d \zeta \geq 0 \quad \text { for all } \quad v_{i}^{n}<z .
$$

Therefore, in such a case, $m\left(\xi ; v_{i-1}^{n}, v_{i}^{n}, v_{i+1}^{n}\right)$ in Lemma 3.3 will be nonnegative if we are able to show that

$$
\begin{array}{cc}
\xi-v_{i}^{n}+\lambda \int_{v_{i}^{n}}^{\xi}\left[a\left(\zeta, v_{i}^{n}, v_{i+1}^{n}\right)-a\left(\zeta, v_{i-1}^{n}, v_{i}^{n}\right)\right] d \zeta \geq 0 & \text { for } \quad v_{i}^{n}<\xi<v_{i}^{n+1}, \\
v_{i}^{n}-\xi-\lambda \int_{\xi}^{v_{i}^{n}}\left[a\left(\zeta, v_{i}^{n}, v_{i+1}^{n}\right)-a\left(\zeta, v_{i-1}^{n}, v_{i}^{n}\right)\right] d \zeta \geq 0 & \text { for } \quad v_{i}^{n+1}<\xi<v_{i}^{n} .
\end{array}
$$

Next, for $u, \bar{v}, \underline{v} \in \mathbb{R}$, let

$$
\bar{u}=u-\lambda(\mathcal{A}(u, \bar{v})-\mathcal{A}(\underline{v}, u)) .
$$

The proof of the theorem is therefore reduced on finding a discrete kinetic flux $a(\xi, u, v)$ such that, 
a) $a(\xi, u, v)$ satisfies the requirements of Proposition 2.6,

b) for any $u, \bar{v}, \underline{v} \in \mathbb{R}$

$$
\begin{gathered}
M(\underline{v}, u, \bar{v})=\xi-u+\lambda \int_{u}^{\xi}[a(\zeta, u, \bar{v})-a(\zeta, \underline{v}, u)] d \zeta \geq 0 \quad \text { for } \quad u<\xi<\bar{u} \\
M(\underline{v}, u, \bar{v})=u-\xi-\lambda \int_{\xi}^{u}[a(\zeta, u, \bar{v})-a(\zeta, \underline{v}, u)] d \zeta \geq 0 \quad \text { for } \quad \bar{u}<\xi<u .
\end{gathered}
$$

In the sequel we show that a discrete kinetic flux that satisfies a) and b) indeed exists. To motivate our construction we will consider first the cases

(I) $u<\xi<\bar{u}, \quad \xi<\{\bar{v}, \underline{v}\}$

(II) $\bar{u}<\xi<u,\{\bar{v}, \underline{v}\}<\xi$.

In case $(\mathrm{I})$ we have $(\mathcal{A}(\xi, u, v)$ is defined in $(2.8))$

$$
\begin{aligned}
M(\underline{v}, u, \bar{v})= & \xi-u+\lambda(\mathcal{A}(\xi, u, \bar{v})-\mathcal{A}(u, u, \bar{v})-\mathcal{A}(\xi, \underline{v}, u)+\mathcal{A}(u, \underline{v}, u)) \\
= & \xi-u+\lambda(\mathcal{A}(\xi, u, \bar{v})-\mathcal{A}(\xi, \underline{v}, u)) \\
= & \frac{1}{2}(\xi-u)+\lambda \mathcal{A}(\xi, u, \bar{v})-\frac{\lambda}{2}[A(u)+A(\xi)] \\
& +\frac{1}{2}(\xi-u)-\lambda \mathcal{A}(\xi, \underline{v}, u)+\frac{\lambda}{2}[A(u)+A(\xi)] .
\end{aligned}
$$

Where we have used that, cf. Proposition 2.6,

$$
\mathcal{A}(u, u, \bar{v})=\mathcal{A}(u, \underline{v}, u)=\int_{-\infty}^{u} a(\zeta) \chi(\zeta, u) d \zeta .
$$

Assume for a moment that $\xi>0$. Then $M(\underline{v}, u, \bar{v})$ is nonnegative if

$$
\begin{aligned}
& \frac{1}{2}(u-\xi)+\frac{\lambda}{2}[A(u)+A(\xi)] \leq \lambda \mathcal{A}(\xi, u, \bar{v}) \\
& \lambda \mathcal{A}(\xi, \underline{v}, u) \leq \frac{1}{2}(\xi-u)+\frac{\lambda}{2}[A(u)+A(\xi)] .
\end{aligned}
$$

Similarly, in the case (II) we have

$$
\begin{aligned}
M(\underline{v}, u, \bar{v})= & u-\xi-\lambda \mathcal{A}(u, u, \bar{v})+\lambda \mathcal{A}(\xi, u, \bar{v})+\lambda \mathcal{A}(u, \underline{v}, u)-\lambda \mathcal{A}(\xi, \underline{v}, u)) \\
= & u-\xi-\lambda \mathcal{A}(u, \bar{v})+\lambda \mathcal{A}(\xi, u, \bar{v})+\lambda \mathcal{A}(\underline{v}, u)-\lambda \mathcal{A}(\xi, \underline{v}, u) \\
= & \frac{1}{2}(u-\xi)-\lambda \mathcal{A}(u, \bar{v})+\lambda \mathcal{A}(\xi, u, \bar{v})+\frac{\lambda}{2}[A(u)-A(\xi)] \\
& +\frac{1}{2}(u-\xi)+\lambda \mathcal{A}(\underline{v}, u)-\lambda \mathcal{A}(\xi, \underline{v}, u)-\frac{\lambda}{2}[A(u)-A(\xi)] .
\end{aligned}
$$

Where we have used that, cf. Proposition 2.6,

$$
\mathcal{A}(u, u, \bar{v})=\mathcal{A}(u, \bar{v})-\int_{u}^{+\infty} a(\zeta) \chi(\zeta, u) d \zeta,
$$


and the similar relation for $\mathcal{A}(u, \underline{v}, u)$. Still assuming $\xi>0$, then $M(\underline{v}, u, \bar{v})$ will be nonnegative if

$$
\begin{aligned}
& \frac{1}{2}(\xi-u)+\lambda \mathcal{A}(u, \bar{v})-\frac{\lambda}{2}[A(u)-A(\xi)] \leq \lambda \mathcal{A}(\xi, u, \bar{v}) \\
& \lambda \mathcal{A}(\xi, \underline{v}, u) \leq \frac{1}{2}(u-\xi)+\lambda \mathcal{A}(\underline{v}, u)-\frac{\lambda}{2}[A(u)-A(\xi)] .
\end{aligned}
$$

Relations (3.9) and (3.10) suggest the following

for $u \leq \xi \leq v: \quad \frac{1}{2}(u-\xi)+\frac{\lambda}{2}[A(u)+A(\xi)] \leq \lambda \mathcal{A}(\xi, u, v) \leq \frac{1}{2}(v-\xi)+\lambda \mathcal{A}(u, v)-\frac{\lambda}{2}[A(v)-A(\xi)]$,

and

for $v \leq \xi \leq u: \quad \frac{1}{2}(\xi-u)+\lambda \mathcal{A}(u, v)-\frac{\lambda}{2}[A(u)-A(\xi)] \leq \lambda \mathcal{A}(\xi, u, v) \leq \frac{1}{2}(\xi-v)+\frac{\lambda}{2}[A(v)+A(\xi)]$.

It will be convenient to introduce the following notation

$$
\begin{aligned}
\lambda \bar{A}_{v>u}(\xi) & =\frac{1}{2}(v-\xi)+\lambda \mathcal{A}(u, v)-\frac{\lambda}{2}[A(v)-A(\xi)] \\
\lambda \underline{A}_{v>u}(\xi) & =\frac{1}{2}(u-\xi)+\frac{\lambda}{2}[A(u)+A(\xi)]
\end{aligned}
$$

and

$$
\begin{aligned}
\lambda \bar{A}_{u>v}(\xi) & =\frac{1}{2}(\xi-v)+\frac{\lambda}{2}[A(v)+A(\xi)] \\
\lambda \underline{A}_{u>v}(\xi) & =\frac{1}{2}(\xi-u)+\lambda \mathcal{A}(u, v)-\frac{\lambda}{2}[A(u)-A(\xi)] .
\end{aligned}
$$

A crucial fact is that, despite the E-property, we have indeed

$$
\underline{A}_{v>u} \leq \bar{A}_{v>u} \text { and } \underline{A}_{u>v} \leq \bar{A}_{u>v} .
$$

This is because by (1.13),

$$
\lambda \bar{A}_{v>u}-\lambda \underline{A}_{v>u}=\frac{1}{2}(v-u)+\lambda \mathcal{A}(u, v)-\frac{\lambda}{2}[A(v)+A(u)]=\frac{1}{2}(v-u)[1-Q(u, v)] \geq 0,
$$

and

$$
\lambda \bar{A}_{u>v}-\lambda \underline{A}_{u>v}=\frac{1}{2}(u-v)-\lambda \mathcal{A}(u, v)+\frac{\lambda}{2}[A(v)+A(u)]=\frac{1}{2}(u-v)[1-Q(u, v)] \geq 0 .
$$

Next, since we are looking for a flux that will satisfy (2.9) and (2.10), it is useful for the sequel to notice that (1.13) implies that for $\xi \in I_{u, v}$

$$
\bar{A}_{v>u}(\xi) \geq \mathcal{A}(u, v), \quad \underline{A}_{v>u}(\xi) \leq A(\xi)
$$

and

$$
\bar{A}_{u>v}(\xi) \geq A(\xi) \quad \underline{A}_{u>v}(\xi) \leq \mathcal{A}(u, v) .
$$


We are ready now to define

$$
\begin{aligned}
& B_{v>u}(\xi)=\min \left\{\bar{A}_{v>u}(\xi), A(\xi)\right\} \quad \text { for } u \leq \xi \leq v \\
& B_{u>v}(\xi)=\min \left\{\bar{A}_{u>v}(\xi), A(u, v)\right\} \quad \text { for } v \leq \xi \leq u
\end{aligned}
$$

Then, since $\mathcal{A}(u, v)$ is an E-flux the above relationships imply,

$$
\mathcal{A}(u, v) \leq B_{v>u}(\xi) \leq A(\xi) \quad \text { and } \quad \underline{A}_{v>u}(\xi) \leq B_{v>u}(\xi) \leq \bar{A}_{v>u}(\xi)
$$

In addition

$$
B_{v>u}(u)=\min \left\{\bar{A}_{v>u}(u), A(u)\right\}=A(u) \text { and } B_{v>u}(v)=\min \{\mathcal{A}(u, v), A(v)\}=A(u, v) .
$$

Where in the first equality in (3.19) we used $\lambda \bar{A}_{v>u}(u)-\lambda A(u)=\frac{1}{2}(v-u)[1-Q(u, v)] \geq 0$. Similar relations hold for $B_{u>v}$. Hence, in the cases under consideration and for $\xi>0$, it suffices to define in $I_{u, v}$,

$$
A(\xi, u, v)= \begin{cases}B_{v>u}(\xi) & \text { for } u \leq \xi \leq v, \xi>0 \\ B_{u>v}(\xi) & \text { for } v \leq \xi \leq u, \xi>0 .\end{cases}
$$

It is clear now that the right extension of $A(\xi, u, v)$ when $\xi<0$ is

$$
A(\xi, u, v)= \begin{cases}B_{v>u}(\xi)-A(\xi) & \text { for } u \leq \xi \leq v, \xi<0 \\ B_{u>v}(\xi)-A(\xi) & \text { for } v \leq \xi \leq u, \quad \xi<0\end{cases}
$$

It is straightforward to see that this choice satisfies a) and b) in the cases (I) and (II) and for $\xi<0$.

In all the other cases the above choice of $A(\xi, u, v)$ satisfies a) and b). Property a) is clear in any case. Property b) is a consequence of (3.18) (and its corresponding relation for $u>v$ ) and of the CFL condition $\lambda \max _{\xi}|a(\xi)| \leq 1$. To illustrate this we consider only the case $\bar{u} \leq \xi \leq u$, $\bar{v}<\xi<0, u<\underline{v}<0$, the other cases being similar. Indeed,

$$
\begin{aligned}
M(\underline{v}, u, \bar{v})= & u-\xi-\lambda \mathcal{A}(u, u, \bar{v})+\lambda \mathcal{A}(\xi, u, \bar{v})+\lambda \int_{\xi}^{u} a(\zeta) \chi(\zeta, u) d \zeta \\
= & u-\xi-\lambda \mathcal{A}(u, \bar{v})+\lambda \int_{u}^{+\infty} a(\zeta) \chi(\zeta, u) d \zeta+\lambda \mathcal{A}(\xi, u, \bar{v}) \\
\geq & \frac{1}{2}(u-\xi)-\frac{\lambda}{2}[A(u)-A(\xi)] \\
& -\lambda A(\xi)+\lambda \int_{u}^{+\infty} a(\zeta) \chi(\zeta, u) d \zeta \\
= & \frac{1}{2}(u-\xi)-\frac{\lambda}{2}[A(u)-A(\xi)]+\lambda[A(u)-A(\xi)] \\
= & \frac{1}{2}(u-\xi)+\frac{\lambda}{2}[A(u)-A(\xi)] \geq 0 .
\end{aligned}
$$

The proof of the kinetic formulation is therefore complete.

The proof of the local entropy inequalities is immediate, again after integrating (1.23) against $S^{\prime}(\xi) d \xi$ 
Remark 3.4. It should be noted that in the proof of the previous theorem the CFL condition (1.13) was used in its local form (only specific $v_{i}^{n}$ appear)

$$
\lambda Q_{i+1 / 2}=\lambda Q\left(v_{i}^{n}, v_{i+1}^{n}\right) \leq 1 .
$$

Indeed, tracing back in the proof we see that $\underline{v}, u$ and $\bar{v}$ represent the values $v_{i-1}^{n}, v_{i}^{n}$ and $v_{i+1}^{n}$.

Remark 3.5. Note that in the semidiscrete case the construction of the Discrete Kinetic Flux is local and therefore the choice of uniform mesh is done only for notational simplicity. In the fully discrete case more care is needed in the construction of the Discrete Kinetic Flux when nonuniform mesh is considered essentially since $a(\xi, u, v)$ depends on $\lambda$. Indeed, denoting by $\lambda^{\prime}$ the CFL number corresponding to the next interval in the construction of the previous theorem, a modification on the choice of $B_{v>u}(\xi), B_{u>v}(\xi)$ is needed depending of the sign of $1-\lambda^{\prime} / \lambda$ : e.g., one may choose

$$
B_{v>u}(\xi)=\left\{\begin{array}{l}
\min \left\{\bar{A}_{v>u}(\xi), A(\xi)\right\} \quad \text { for } u \leq \xi \leq v \quad \text { provided } \lambda \geq \lambda^{\prime} \\
\max \left\{\underline{A}_{v>u}(\xi), A(u, v)\right\} \quad \text { for } u \leq \xi \leq v \quad \text { provided } \lambda^{\prime} \geq \lambda
\end{array}\right.
$$

\section{Engquist-Osher scheme}

\subsection{Semidiscrete Engquist-Osher scheme}

We give first a direct proof for the kinetic formulation of the Engquist-Osher scheme [7]. We also give explicit formulas for the kinetic defect measures $m_{ \pm}$.

Theorem 4.1. There is a unique nonnegative function $m_{i}(t, \xi)$ with bounded support in $\xi$ such that the scheme (1.4) with Engquist-Osher flux (1.21) is equivalent to the kinetic equation (1.17)(1.18), with $a_{ \pm}(\xi, u, v)=a_{ \pm}(\xi)$. Moreover, we have the bound

$$
\sum_{i \in \mathbb{Z}} h \int_{0}^{\infty} m_{i}(t, \xi) d t \leq \mathbb{I}_{\{\xi \geq 0\}}\left\|\left(v_{i}^{0}-\xi\right)_{+}\right\|_{l^{1}}+\mathbb{I}_{\{\xi \leq 0\}}\left\|\left(\xi-v^{0}\right)_{+}\right\|_{l^{1}} \leq\left\|v^{0}\right\|_{l^{1}},
$$

and the function $m_{ \pm}$are given by

$$
\begin{gathered}
m_{+}(\xi ; u, v)=A_{-}(u)-A_{-}(\xi), \quad \text { for } u \leq \xi \leq v, \\
m_{+}(\xi ; u, v)=A_{-}(v)-A_{-}(\xi), \quad \text { for } v \leq \xi \leq u, \\
m_{-}(\xi ; u, v)=A_{+}(\xi)-A_{+}(u), \quad \text { for } u \leq \xi \leq v, \\
m_{-}(\xi ; u, v)=A_{+}(\xi)-A_{+}(v), \quad \text { for } v \leq \xi \leq u .
\end{gathered}
$$

In other words, the Engquist-Osher scheme is nothing but a linear upwind discretization of the kinetic formulation. Also Theorem 2.1 (ii) implies that the EO scheme satisfies all local entropy inequalities. 
Proof. As in Lemma 2.1 we see that

$$
m_{i}(t, \xi)=\left[m_{+}\left(\xi ; v_{i}(t), v_{i+1}(t)\right)+m_{-}\left(\xi ; v_{i-1}(t), v_{i}(t)\right)\right]
$$

where the functions $m_{+}$satisfy

$$
\begin{gathered}
\frac{\partial}{\partial \xi} m_{+}\left(\xi ; v_{i}(t), v_{i+1}(t)\right)= \\
\delta\left(\xi-v_{i}(t)\right)\left[A\left(v_{i}(t)\right)-A_{i+1 / 2}\right]+\left[a_{+}(\xi) \chi\left(\xi, v_{i}(t)\right)-a_{-}(\xi) \chi\left(\xi, v_{i+1}(t)\right)\right]-a(\xi) \chi\left(\xi, v_{i}(t)\right)
\end{gathered}
$$

and

$$
\begin{gathered}
\frac{\partial}{\partial \xi} m_{-}\left(\xi ; v_{i-1}(t), v_{i}(t)\right)= \\
\delta\left(\xi-v_{i}(t)\right)\left[-A_{i-1 / 2}-A\left(v_{i}(t)\right)\right]-\left[a_{+}(\xi) \chi\left(\xi, v_{i-1}(t)\right)-a_{-}(\xi) \chi\left(\xi, v_{i}(t)\right)\right]+a(\xi) \chi\left(\xi, v_{i}(t)\right) .
\end{gathered}
$$

Then, with a slight change of notation,

$$
\begin{gathered}
\frac{\partial}{\partial \xi} m_{+}(\xi ; u, v)= \\
=-\delta(\xi-u) \int_{\mathbb{R}} a_{-}(\zeta)[\chi(\zeta ; u)-\chi(\zeta ; v)] d \zeta+a_{-}(\xi)[\chi(\xi ; u)-\chi(\xi ; v)] .
\end{gathered}
$$

Notice that the integral in $\xi$ of the righthand side of this idendity vanishes. Therefore since $m_{+}$ should have compact support in $\xi$,

$$
m_{+}(\xi ; u, v)=0 \quad \text { for } \xi \notin[u, v] \quad \text { (non - ordered interval). }
$$

Indeed, the brackets $[\chi(\zeta ; u)-\chi(\zeta ; v)]$ and $[\chi(\xi ; u)-\chi(\xi ; v)]$ are supported in $[u, v]$. Also, they have the same sign as $u-v$. Therefore, either $v<u$ and $\frac{\partial}{\partial \xi} m_{+}(\xi ; u, v)$ is positive beetween $v$ and $u$ and thus $m_{+}(\xi ; u, v)$ vanishes for $\left.\left.\xi \in\right]-\infty, v\right]$, is nonnegative for $\xi \in[v, u]$ and has a jump at $\xi=u$, vanishes for $\xi>u$. Either $v>u$, and a similar argument shows that $m_{+}(\xi ; u, v)$ is again nonnegative. The same argument as before shows that $m_{-}(\xi ; u, v)$ is nonnegative also.

Finally, the bound on the discrete kinetic defect measure is obtained as in the continous case. We first argue for $\xi_{0} \geq 0$. We use Kruzkov's entropy $S_{\xi_{0}}^{+}(\xi)=\left(\xi-\xi_{0}\right)_{+}, S^{+\prime \prime}(\xi)_{\xi_{0}}=\delta\left(\xi=\xi_{0}\right)$, and multiply the equation (1.17) by $S_{\xi_{0}}^{+\prime}(\xi)$, integrate in $\xi$, in time and sum up on $i$. Taking into account the sign in the quantity

$$
\int_{\mathbb{R}} S_{\xi_{0}}^{+\prime}(\xi) \chi\left(\xi ; v_{i}(t)\right) d \xi \geq 0
$$

we obtain the inequality, for $\xi_{0} \geq 0$,

$$
\begin{gathered}
\sum_{i \in \mathbb{Z}} h \int_{0}^{\infty} m_{i}\left(t, \xi_{0}\right) d t \leq \sum_{i \in \mathbb{Z}} h\left\|\left(v_{i}^{0}-\xi\right)_{+}\right\|_{l^{1}} \\
\leq\left\|v^{0}\right\|_{l^{1}} .
\end{gathered}
$$

A similar argument for $\xi_{0} \leq 0$ concludes the proof of the proposition. 


\subsection{Fully discrete Engquist-Osher scheme}

As in Section 3, we depart from the fully discrete Engquist-Osher scheme (1.5), and define $f_{i}^{n+1}(\xi)$ by the formula

$$
\begin{aligned}
f_{i}^{n+1}(\xi)-\chi\left(\xi, v_{i}^{n}\right) & +\lambda\left[a_{+}(\xi) \chi\left(\xi, v_{i}^{n}\right)-a_{-}(\xi) \chi\left(\xi, v_{i+1}^{n}\right)\right] \\
& -\lambda\left[a_{+}(\xi) \chi\left(\xi, v_{i-1}^{n}\right)-a_{-}(\xi) \chi\left(\xi, v_{i}^{n}\right)\right]=0 .
\end{aligned}
$$

which can also be written under the kinetic form

$$
\begin{aligned}
\chi\left(\xi, u_{i}^{n+1}\right)-\chi\left(\xi, v_{i}^{n}\right) & +\lambda\left[a_{+}(\xi) \chi\left(\xi, v_{i}^{n}\right)-a_{-}(\xi) \chi\left(\xi, v_{i+1}^{n}\right)\right] \\
& -\lambda\left[a_{+}(\xi) \chi\left(\xi, v_{i-1}^{n}\right)-a_{-}(\xi) \chi\left(\xi, v_{i}^{n}\right)\right]=\frac{\partial}{\partial \xi} m_{i}^{n}(\xi)
\end{aligned}
$$

where $m_{i}^{n}(\xi)$ vanishes at $\pm \infty$ because

$$
u_{i}^{n+1}=\int_{\mathbb{R}} f_{i}^{n+1}(\xi) d \xi
$$

We claim that for $\lambda$ small enough this is a kinetic formulation.

Theorem 4.2. Consider the scheme (1.5), (1.21), and assume the CFL condition

$$
\lambda \max _{\xi}|a(\xi)| \leq 1
$$

then (4.3) holds with $m_{i}^{n}(\xi) \geq 0$ satisfying (1.18), and thus it is a kinetic formulation of EngquistOsher scheme.

Proof. Using (4.4), and a variant of Brenier's lemma ([4] or [16] Ch. 2.2), the property

$$
0 \leq \operatorname{sgn}(\xi) f_{i}^{n+1}(\xi) \leq 1
$$

is enough to ensure that $m_{i}^{n}(\xi) \geq 0$, using the relation

$$
\frac{\partial}{\partial \xi} m_{i}^{n}(\xi)=\chi\left(\xi, u_{i}^{n+1}\right)-f_{i}^{n+1}(\xi)
$$

To check the signs in (4.6), we rewrite (4.2) as

$$
\begin{aligned}
f_{i}^{n+1}(\xi)= & \chi\left(\xi, v_{i}^{n}\right)\left(1-\lambda a_{+}(\xi)-\lambda a_{-}(\xi)\right) \\
& +\lambda \chi\left(\xi, v_{i+1}^{n}\right) a_{-}(\xi)+\lambda \chi\left(\xi, v_{i+1}^{n}\right) a_{+}(\xi) .
\end{aligned}
$$

To prove the property (4.6), it is enough to notice that this is a convex combination of $\chi$ 's, a property which follows obviously from $a_{ \pm} \geq 0$ and (4.5).

Acknowledgment. The authors would like to thank the referees of this paper for their useful comments. 


\section{References}

[1] S. Benharbit, A.Chalabi and J.-P. Vila, Numerical viscosity and convergence of finite volume methods for conservation laws with boundary conditions. SIAM J. Numer. Anal. 32 (1995) 775-796

[2] R. Botchorishvili, B. Perthame and A. Vasseur, Eqilibrium Schemes for Scalar Conservation Laws with Stiff Sources. INRIA Report RR-3891 (2000), Math. Comp., to appear.

[3] F. Bouchut, Construction of BGK models with a family of kinetic entropies for a given system of conservation laws, J. Stat. Phys. 95 (1999) 113-170.

[4] Y. Brenier, Résolution d'équations d'évolution quasilinéaires en dimensions $\mathrm{N}$ d'espace à l'aide d'équations linéaires en dimensions N+1, J. Diff. Eq. 50(3) (1982) 375-390.

[5] B. Cockburn, F. Coquel, P.G. LeFloch, An error estimate for finite volume methods for multidimensional conservation laws, Math. Comp. 63 (1994) 77-103

[6] C.M. Dafermos, Hyperbolic conservation laws in continuum physics, Springer Verlag, GM 325, 1999.

[7] B. Enquist and S. Osher, Stable and entropy satisfying approximations for transonic flow calculations, Math. Comp. 31 (1980) 45-75.

[8] R. Eymard, T. Gallouët and R. Herbin, Finite volume methods, In Handboook of numerical analysis, vol. VII, P.G. Ciarlet and J-L. Lions eds., North-Holland, Amsterdam, 2000.

[9] E. Godlewski, P.A. Raviart Hyperbolic systems of conservation laws, Ellipses, 1991.

[10] D. Kröner, Numerical schemes for conservation laws. Wiley and Teubner, 1997.

[11] R.J. LeVeque, Numerical Methods for Conservation Laws, Lectures in Methematics, ETH Zurich, Birkhauser, 1992.

[12] P-L. Lions, B. Perthame and E. Tadmor, Formulation cinétique des lois de conservation scalaires multidimensionnelles C. R. Acad. Sci. Paris Serie I 312 (1991) 97-102.

[13] P-L. Lions, B. Perthame and E. Tadmor, A kinetic formulation of multidimensional scalar conservation J. Amer. Math. Soc. 7 (1994) 169-191.

[14] S. Osher, Riemann solvers, the entropy condition and numerical schemes, S.I.A.M. J. Numer. Anal. 21 (1984) 217-235

[15] S. Noelle, Convergence of higher order finite volume schemes on irregular grids, Advances in Comput. Math. 3 (1995)

[16] B. Perthame, Kinetic Formulations of Conservation Laws, Oxford University Press, 2002.

[17] D. Serre, Systèmes hyperboliques de lois de conservation, Parties I et II. Diderot, Paris, 1996.

[18] E. Tadmor Numerical viscosity and the entropy condition for conservative difference schemes, Math. Comp. 43 (1984) 369-381.

[19] A. Vasseur, Convergence of a semi-discrete kinetic scheme for the system of isentropic gas dynamics with $\gamma=3$. Indiana Univ. Math. J. 48 (1999), no. 1, 347-364.

[20] A. Vasseur, Kinetic semidiscretization of scalar conservation laws and convergence by using averaging lemmas. SIAM J. Numer. Anal. 36 (1999), no. 2, 465-474.

[21] M. Westdickenberg and S. Noelle, A new convergence proof for finite volume schemes using the kinetic formulation of conservation laws, S.I.A.M. J. Numer. Anal. 371 (2000) 742-757. 